\title{
Article
}

\section{Developing the hypoglycemia-nursing early warning score system (Hy-NEWSS): A pilot study}

\author{
Ahmad Hasyim Wibisono, ${ }^{1}$ Dennti Kurniasih, ${ }^{2}$ Eti Sumartiyah, ${ }^{3}$ Rima Novia Putri, ${ }^{4}$ \\ Dikha Ayu Kurnia, ${ }^{5}$ Yunisar Gultom, ${ }^{3}$ Debie Dahlia ${ }^{5}$
}

${ }^{1}$ School of Nursing, Faculty of Medicine, Universitas Brawijaya, Malang; ${ }^{2}$ RSUD Jenderal Ahmad Yani Metro Lampung; ${ }^{3}$ Nursing Department, RSUPN Dr Cipto Mangunkusumo, Jakarta; ${ }^{4}$ Nursing Department, State Health Polytechnic, Tanjung Pinang; ${ }^{5}$ Faculty of Nursing, University of Indonesia, Depok, Indonesia

\begin{abstract}
Background: Severe hypoglycemia occurs in $6 \%$ of type 2 DM patients with a 6 to $7 \%$ mortality rate. Amidst the COVID-19 pandemic, there has been a higher rate of fatality among diabetes patients, at least $22 \%$ of COVID-19 deaths in Wuhan, China. Furthermore, the condition impairs physiological defense against recurrent hypoglycemia in the future. Accordingly, the innovation in this study aims to prevent severe hypoglycemia among type 2 patients through early risk screening, the use of preventative protocols, and patient and family empowerment. This intervention bundle is called Hypoglycemia-Nursing Early Warning Score System (Hy-NEWSS).

Design and Methods: The innovation was implemented in inpatient and outpatient settings, within two weeks with the following sequence: i) internal training, ii) Hy-NEWSS implementation, iii) outcomes evaluation.

Results: Of the twenty-eight patients involved in the study, 3 patients $(19 \%)$ had a high risk of hypoglycemia, while the remaining had a low risk for the condition. However, at the end of the study there was no event of severe hypoglycemia $(0 \%)$, and only 1 event $(6.33 \%)$ of mild hypoglycemia. The results also showed that the accuracy of nurses in implementing the protocols was $93 \%$ and that during the evaluation, $95.62 \%$ of the responses of the nurses from the inpatient and outpatient settings were positive. Finally, the scores of the sensitivity and specificity evaluation were $99 \%$ and $88 \%$, respectively.

Conclusion: It may be concluded from this initial study that Hy-NEWSS is effective in preventing severe hypoglycemia, accurate in identifying patient risk level for the condition, and has high applicability to be implemented in hospital settings.
\end{abstract}

\section{Introduction}

Severe hypoglycemia ( $\mathrm{SH}$ ) is estimated to occur in $6 \%$ of patients with type $2 \mathrm{DM}$ and has a mortality rate of $6 \%$ to $7 \%{ }^{1}$ Furthermore, its incidence increases the likelihood for patients to experience hypoglycemia associated autonomic failure (HAAF). 2,3 During the COVID-19 pandemic, there has been a higher rate of fatality among diabetes patients. ${ }^{4}$ Hypoglycemia is one of the most common complications of diabetes, especially in those undertaking insulin therapy. ${ }^{5,6} \mathrm{~A}$ study in Wuhan, China revealed that at least $22 \%$ of COVID-19 deaths are related to the disease. ${ }^{7}$ Based on the 4 months of clinical observation in one of the type A hospitals in Jakarta, Indonesia, it was concluded that hypoglycemia is one of the common cause of hospitalization of type 2 DM (T2DM) patients, along with diabetic ketoacidosis (DKA), hyperosmolar hyperglycemic syndrome (HHS), and recurrent ulceration. However, $\mathrm{SH}$ was chosen as the central issue because there was a period when it occurred 4 times during 1 certain month of observation. Thus, there was an average of 1 incidence per week. Following the above findings, an internal discussion was conducted involving the nurse managers, the nurse in charge, and the nursing staffs to further analyze the local circumstances using the strengths, weaknesses, opportunities, and threats (SWOT) method. The analysis result showed that $\mathrm{SH}$ was one of the most important issues in T2DM patient care. It also showed that the hospital had sufficient resources to overcome the SH problem. Therefore, a clinical innovation through protocol development needs to be established to tackle the occurrence of this problem, especially in inpatient settings.

As a basis for the protocol development, a task force was appointed to collect and analyze recent publications, guidelines, and evidence on $\mathrm{SH}$ prevention. After several weeks of literature exploration, the team was unable to find any particular guideline on SH prevention in hospital settings from several online scientific databases. Therefore, a certain protocol needs to be developed as a preventative measure for $\mathrm{SH}$ in hospital settings.

Based on the situation above, this project was then named hypoglycemia-nursing early warning score system (Hy-NEWSS). Meanwhile, this is an early warning system that would complement the pre-existing general nursing early warning system. In the initial stage, the Hy-NEWSS project had the following objectives: i) to develop, apply, and evaluate the $\mathrm{SH}$ risk screening tool, ii) to develop, apply, and evaluate intervention protocols to prevent the occurrence of SH.

Significance for public health

Hypoglycemia is a deteriorating problem and affects type 2 diabetes patients in both hospital and community settings. Furthermore, its incidence was reported to be significantly higher in patients undertaking insulin therapy, especially those suffering from other chronic comorbidities. Currently, there is no standardized prevention protocol for hypoglycemia. Therefore, this study describes an initial effort in developing an evidence-based intervention bundle to prevent the condition. 


\section{Design and Methods}

This project was conducted in a multistage process, where different methods were used depending on the aim of each stage. The breakdown of each stage and the corresponding methods is provided in Table 1. In general, there were three stages as follows:

1. Pre-clinical preparation

The objective of this stage is to develop a stratification tool for classifying SH risk, and to develop a protocol to manage each risk level. Such objectives were achieved through systematic review of the current literature.

2. Clinical study (pilot project)

In this stage, the objectives were:

- To measure the sensitivity and specificity of the risk stratification tool.

- To measure the effectiveness of the SH prevention protocols.

- To obtain nurses' perceptions about the clinical relevance of the project.

These objectives were achieved using a quasi-experimental, with a prospective cohort method. Participants were nurses in the inpatient setting, internal medicine ward.

3. Data analysis

This stage was aimed to analyze the data gathered from the clinical trial, both from patients' outcomes and the nurses' perspectives on the clinical visibility of the project.

The study was conducted in one of the type A hospitals in Jakarta, Indonesia. Moreover, this hospital is a referral hospital and receives patients from lower types of hospitals. Thus, most of the patients in this hospital have multiple comorbidities and significant complications. With these conditions, patients are at risk of developing severe hypoglycemia. Participants were categorized into two groups, patient-participants and nurse-participants. The patient participants were adults with T2DM, while the nurse participants were nurses working in the inpatient diabetes care unit.

\section{Results and Discussions}

This study involved 20 nursing staff, and the intervention was performed on 28 patients. Due to the outbreak of the covid-19 pandemic, the implementation period of this study was unexpectedly reduced to only seven days. However, the 7 days of implementation was still able to provide some insights about the Hy-NEWSS project. The patients were of equal proportion in terms of gender, and each of them suffered from various T2DM complications including retinopathy, foot ulceration, end-stage renal disease, and peripheral neuropathy.

\section{The risk stratification tool}

There were very limited numbers of $\mathrm{SH}$ risk stratification tools. Also, the systematic review only found 2 articles addressing this issue, the first one by Karter et al., ${ }^{8}$ and the one developed by Han et al. ${ }^{9}$ was selected as the most relevant to this project. One of the main advantages of the tool used in this study is that it incorporates comorbid conditions as one of the determining factors of SH risk.

At the initial screening, 4 patients $(14.29 \%)$ were identified as having a high risk of $\mathrm{SH}$, while the remaining $85.71 \%$ had a low risk. The screening itself was conducted daily for each patient in order to anticipate the dynamics and changes in the conditions of each of them. Finally, the risk levels were subject to corresponding preventive protocol. At the end of the intervention, it was ascertained that the sensitivity of the risk stratification tool was $99 \%$, while the specificity was $88 \%$. Furthermore, the positive and negative predictive values were 24 and $99 \%$, respectively. From the nurses' perspective, the use of hypoglycemia risk stratification tool was perceived as a favorable intervention. As indicated in the Table $2,100 \%$ of the respondents agreed or strongly agreed that it is clinically beneficial for patients and need to be performed on a regular basis. The availability of the stratification tool as a digital platform was also found easy to use and does not increase nurse workload as indicate by $75 \%$ and $70 \%$ of the respondents respectively.

Table 1. Breakdown of the study stages and methods.

\begin{tabular}{|c|c|c|c|}
\hline Stage & Aim & Methods & Timeframe \\
\hline Preclinical Preparation & $\begin{array}{l}\text { To develop a stratification tool for SH risk level } \\
\text { To develop an intervention protocol for each risk level }\end{array}$ & $\begin{array}{l}\text { Systematic review } \\
\text { Systematic review }\end{array}$ & 6 weeks \\
\hline Clinical study (pilot project) & $\begin{array}{l}\text { To measure the sensitivity and specificity of the risk stratification tool } \\
\text { To measure the effectiveness of the SH prevention protocols } \\
\text { To obtain nurses' perceptions about the clinical relevance of the project }\end{array}$ & $\begin{array}{l}\text { Cohort study } \\
\text { Cross-sectional study }\end{array}$ & 4 weeks \\
\hline Data analysis & To evaluate the results of the clinical study stage & Statistical analysis & 1 week \\
\hline
\end{tabular}

SH, severe hypoglycemia.

Table 2. Nurse participants' perceptions on SH risk stratification tool.

This SH risk stratification tool is:

Clinically beneficial for patients

Suitable for hospital use

Needs to be performed on a regular basis

Does not increase nurses' workloads

Easy to perform

A significant part of nursing documentation

In line with nurses' clinical authority

Suitable to be included as a standard procedure for the care of T2DM patients

SH, severe hypoglycemia; T2DM, type 2 diabetes mellitus.

\begin{tabular}{cccc}
0 & 0 & $16(80 \%)$ & $4(20 \%)$ \\
$1(5 \%)$ & 0 & $17(85 \%)$ & $2(10 \%)$ \\
0 & 0 & $17(85 \%)$ & $3(15 \%)$ \\
$1(5 \%)$ & $5(25 \%)$ & $13(65 \%)$ & $1(5 \%)$ \\
$1(5 \%)$ & $4(20 \%)$ & $12(60 \%)$ & $3(15 \%)$ \\
0 & 0 & $12(60 \%)$ & $8(40 \%)$ \\
0 & 0 & $15(75 \%)$ & $5(25 \%)$ \\
0 & 0 & $14(70 \%)$ & $6(30 \%)$ \\
\hline
\end{tabular}




\section{The hypoglycemia prevention protocols}

A particular intervention bundle was developed for each $\mathrm{SH}$ risk level. Furthermore, as previously described, the levels were categorized as high risk and low risk with the cut-off point being $6 \%$. For each risk level, the preventive protocol includes an observation guideline, a particular procedure, and a multidisciplinary team. The intervention bundles were extracted from a large number of references including the American Diabetes Association, ${ }^{10}$ the Indonesian Society of endocrinology, ${ }^{11}$ Seaquist et al., ${ }^{12}$ Riddle et al., ${ }^{13}$ Evert et al., ${ }^{14}$ and Yale et al. ${ }^{3}$

During the intervention period, none of the patients with a high risk of $\mathrm{SH}$ experienced the condition, which means that the incidence of SH was $0(0 \%)$ event. Also, only $1(3.5 \%)$ patient experienced mild hypoglycemia. In addition to patients' clinical parameters, this study also evaluated the relevance of the intervention bundle to the hospital setting and the nurses' perspective on how the Hy-NEWSS could improve the nursing practice. Among the patient participants, 27 (93\%) received the protocols appropriately, while only $2(7 \%)$ received them inappropriately. This indicated that the nurses were able to apply the protocols appropriately, and may indicate that the protocols were easy to apply.

The hypoglycemia risk stratification tool received $92.5 \%$ of favorable responses from the nurse participants, which means that the tool was deemed as clinically relevant to be applied to patients in the hospital setting. Similarly, the hypoglycemia prevention protocols received $98.12 \%$ of favorable responses, which also means that it was highly relevant to be applied. Finally, from the overall perspective, the Hy-NEWSS intervention bundle received $96.25 \%$ of favorable responses. Tables 2, 3, and 4 illustrate how nurse participants perceive the clinical relevance of each tool and the overall project.

The first thing to highlight in this study was the zero incidence of SH during the Hy-NEWSS implementation, even though there were some patient participants with high risk for the condition. This may indicate that the intervention bundle was both accurate and effective in preventing SH. Every aspect of the Hy-NEWSS was built using the latest evidence, incorporated multi-modality interventions, and used a multidisciplinary approach, with a patient-centered care paradigm. Patients and families were also given certain roles during Hy-NEWSS implementation. This is because as stated by the IDF (2012) in its global guideline, ideally T2DM management involves a multidisciplinary team. ${ }^{15}$ In addition, patients and families have to be put at the center of the circle to manage their conditions themselves with the assistance of the healthcare team. ${ }^{16}$ Patient and family education were one of the cornerstones in achieving diabetes control goals. ${ }^{17}$ This is because a combination of adequate knowledge and patient empowerment increases the likelihood of patients achieving diabetes control targets. ${ }^{18}$ In a large scale-referral hospital, limited manpower often becomes a recurring problem, especially in developing countries. This condition even became worse amidst the COVID-19 pandemic because of the number of healthcare professionals that are used in COVID-19 treatment units. Therefore, patient and family activation may help to partially solve the manpower issue and contribute towards a better clinical outcome. In this project, patient and family education sessions were conducted in a gradual and stepwise fashion. Furthermore, patients and families were equipped with a handbook that contained information needed to perform their roles during the Hy-NEWSS implementation.

As stated by LaManna et al., effective patient-centered diabetes education contributes significantly in preventing hypoglycemia, both during hospitalization and following hospital discharge. ${ }^{19}$ This could be achieved through an effective skill acquisition by patients and families on the detection of early signs of hypoglycemia, the proper response to the condition, and the appropriate use of emergency medical services. ${ }^{20}$ By doing so, the task of taking care of patients would be shared between healthcare professionals and families, and thus, there would adequate monitoring and care for T2DM patients with high or low risk of SH. This could then cause a reduction in the rate of hypoglycemia-related morbidity and mortality. At the end of the implementation stage, a visibil-

Table 3. Nurses' perceptions on $\mathrm{SH}$ prevention protocols.

\begin{tabular}{|c|c|c|c|c|}
\hline Evaluation item & Strongly disagree & Disagree & Agree & Strongly agree \\
\hline
\end{tabular}

SH, severe hypoglycemia; T2DM, type 2 diabetes mellitus.

Table 4. Nurses' perceptions on the overall project.

\begin{tabular}{|c|c|c|c|c|}
\hline Evaluation item & Strongly disagree & Disagree & Agree & Strongly agree \\
\hline \multicolumn{5}{|l|}{ This Hy-NEWSS intervention bundle is: } \\
\hline Clinically beneficial for patients & 0 & 0 & $15(75 \%)$ & $5(25 \%)$ \\
\hline Suitable for hospital use & 0 & 0 & $15(75 \%)$ & $5(25 \%)$ \\
\hline Suitable for a further process into formal regulation & 0 & 0 & $15(75 \%)$ & $5(25 \%)$ \\
\hline Does not increase nurses' workloads & 0 & $6(30 \%)$ & $12(60 \%)$ & $2(10 \%)$ \\
\hline Improves professional nursing practice & 0 & 0 & $14(70 \%)$ & $6(30 \%)$ \\
\hline In line with nurses' clinical authority & 0 & 0 & $14(70 \%)$ & $6(30 \%)$ \\
\hline
\end{tabular}

Hy-NEWSS, hypoglycemia-nursing early warning score system. 
ity study was conducted to examine whether the Hy-NEWSS was considered as a clinically visible intervention or not. The parameters that were looked at are nurses' perceptions of several aspects as follows: i) clinical benefit for patients, ii) impact on workload, iii) relevance to hospital settings, iv) urgency/clinical importance, and $\mathrm{v}$ ) relevance to professional nursing practice.

It was quite surprising that during the COVID-19 pandemic the Hy-NEWSS intervention bundle was considered a highly favorable task, as most of the nurse participants' responses were either on the "agree" or "strongly agree" score.

\section{The risk stratification tool's accuracy}

The analysis on specificity (Sp) and sensitivity ( $\mathrm{Sn}$ ) showed high scores of $88 \%$ and $99 \%$ respectively. These numbers were higher than those of the original study by Han et al. ${ }^{9}$ The high $\mathrm{Sn}$ score indicated that the HY-NEWSS was very accurate in predicting the likelihood of T2DM patients to experience hypoglycemia. Meanwhile, the high Sp score indicated that Hy-NEWSS was very accurate in distinguishing those with a low possibility for the condition. Interesting findings were exhibited by the predictive values of the tool. The positive predictive value (PPV) was relatively low $(25 \%)$ which means that only $25 \%$ of the predicted patients were actually experiencing hypoglycemia. This may indicate the effectiveness of the preventative protocols, as they reduced the occurrence of hypoglycemia by $75 \%$. On the other hand, the negative predictive value (NPV) value was very high (99\%) which means that the prediction on the non-occurrence of hypoglycemia was actually relevant in reality. Both of these scores correspond to support the clinical effectiveness of the protocols to prevent the incidence of hypoglycemia.

\section{Implications for nursing practice and public health}

The Hy-NEWSS was aimed at preventing the occurrence of $\mathrm{SH}$ in patients with T2DM in hospital settings. This intervention bundle is an important preventive measure and is highly relevant in the context of nursing practice. As reflected in the nursing diagnosis taxonomy in domain 4 and diagnosis class metabolism, one of the responses of patients towards T2DM is the exhibition of unstable blood glucose level. ${ }^{21}$ Furthermore, in the nursing interventions classification, hypoglycemia is one of the nursing interventions as reflected by the intervention label of hypoglycemia management, which consists of preventative interventions of hypoglycemia. ${ }^{22}$ From the aforementioned explanation, the linkage between Hy-NEWSS and the nursing process is clearly visible.

From the human adaptation system perspective, as postulated by Roy in the Roy Adaptation Model, the Hy-NEWSS is a representation of nursing care, which aims to facilitate patients to gain adaptive response towards T2DM and prevent maladaptive/ineffective responses. ${ }^{23}$ The Hy-NEWSS interventions bundle provides adaptation support on regulator and cognator subsystems. The regulator and cognator are parts of the Roy's adaptation model which serve as effector of human system in response to external stimuli. ${ }^{24}$ Meanwhile, family empowerment in Hy-NEWSS represents the adaptive facilitation on the interdependence mode.

In the Hy-NEWSS intervention bundle, nurses were actively involved in patient care and took part in a multidisciplinary team, which also involved physicians and dieticians, because the protocols were developed using a multidisciplinary team perspective. Besides, the protocols were developed to fall in-line with the global diabetes plan of the International Diabetes Federation (IDF), in which it is stated that a multidisciplinary-well coordinated healthcare team is essential to achieve clinical parameters. In that document, the IDF also stated that the collaboration needs to also involve the management and decision makers. ${ }^{25}$
As indicated in the table 4, the overall intervention bundle was perceived as suitable for hospital use, clinically beneficial for patients, improves professional nursing practice, and is recommended for further process into formal regulation as it received $100 \%$ favorable response (agree or strongly agree) from the respondents on those items. Furthermore, a majority of respondents $(70 \%)$ perceived the Hy-NEWSS does not increase nurse workload, and $100 \%$ of respondents perceived the Hy-NEWSS is in line with nurses' clinical authority.

Finally, the Hy-NEWSS also contains clinical novelty and represent the role of clinical nurse specialists in patient care. As stated by Han et al. and Karter et al., recently the hypoglycemia prevention protocols are scattered and locally developed. However, none has been identified as an evidence-based protocol, and clinically tested to become a guideline or best practice. Similarly, the SH risk stratification protocol is also difficult to find in the online research databases. ${ }^{8,9}$

\section{Conclusions}

From this initial study, it may be concluded that the risk stratification tool has been proven as both sensitive and specific for determining the risk of SH occurring. Further, the SH prevention protocols are effective in reducing the incidence of $\mathrm{SH}$ as indicated by the predictive value scores.

Correspondence: Ahmad Hasyim Wibisono, School of Nursing, Faculty of Medicine, Universitas Brawijaya, Jl. Puncak Dieng, Kunci, Kalisongo, Kec. Dau, Malang, East Java Indonesia 65151.

Tel. +62.341569117 - Fax: +62.341564755.

E-mail: ahmad.wibisono@ub.ac.id

Key words: Severe hypoglycemia; early screening; preventative protocols; patient empowerment.

Contributions: All authors contributed equally to this article, while DD, DAK, and YG served as supervisors and reviewed the final article.

Conflict of interest: The author declares that there is no potential conflict of interest.

Funding: This study was partially supported financially by the School of Nursing, Brawijaya University, Malang, Indonesia.

Acknowledgments: The authors are grateful to INHSS 2020 committee from the School of Nursing, Faculty of Medicine, Universitas Brawijaya, Malang, Indonesia, and to the reviewer board.

Ethics approval: This study was approved by the Health Research Ethics Committee of the Faculty of Nursing, University of Indonesia.

Conference presentation: Part of this study was presented at the $1^{\text {st }}$ International Nursing and Health Sciences Symposium, November $13^{\text {th }}$ to $15^{\text {th }} 2020$, Brawijaya University, Malang, Indonesia.

Received for publication: 18 January 2021.

Accepted for publication: 10 March 2021.

o Copyright: the Author(s), 2021

Licensee PAGEPress, Italy

Journal of Public Health Research 2021;10:2145

doi:10.4081/jphr.2021.2145

This work is licensed under a Creative Commons Attribution NonCommercial 4.0 License (CC BY-NC 4.0). 


\section{References}

1. Edridge CL, Dunkley AJ, Bodicoat DH, et al. Prevalence and incidence of hypoglycaemia in 532, 542 people with type 2 diabetes on oral therapies and insulin: A systematic review and meta-analysis of population based studies. PLoS One 2015; 10:e0126427.

2. Fauci S, Kasper D, Hauser S, et al. Harrison's principles of internal medicine. 19th ed. Michigan: McGraw-Hill Education; 2014.

3. Clayton D, Woo V, Yale JF. Hypoglycemia. Can J Diabetes 2013;37:S69-71.

4. Hussain A, Bhowmik B, do Vale Moreira NC. COVID-19 and diabetes: Knowledge in progress. Diabetes Res Clin Pract 2020;162:.108142.

5. Cho NH, Shaw JE, Karuranga S, et al. IDF diabetes atlas: Global estimates of diabetes prevalence for 2017 and projections for 2045. Diabetes Res Clin Pract 2018;138:271-81.

6. Olveira G, Abuín J, López R, et al. Risk factors for hypoglycemia in inpatients with total parenteral nutrition and type 2 diabetes: A post hoc analysis of the InSUPAR study. Endocr Pract 2020;26:604-11.

7. Angelidi AM, Belanger MJ, Mantzoros CS. COVID-19 and diabetes mellitus: What we know, how our patients should be treated now, and what should happen next. Metabolism 2020;107:154245.

8. Karter AJ, Warton EM, Lipska KJ, et al. Development and validation of a tool to identify patients with type 2 diabetes at high risk of hypoglycemia-related emergency department or hospital use. JAMA Intern Med 2017;177:1461-70.

9. Han K, Yun JS, Park YM, et al. Development and validation of a risk prediction model for severe hypoglycemia in adult patients with type 2 diabetes: A nationwide population-based cohort study. Clin Epidemiol 2018;10:1545-59.

10. American Diabetes Association [Internet]. Hypoglycemia (low blood glucose). American Diabetes Association. 2020 [cited 2020 Oct 1]. Available from: https://www.diabetes.org/healthy-living/medication-treatments/blood-glucose-testing-and-control/hypoglycemia

11. Rudianto AD. [Konsensus Pengelolaan dan Pencegahan Diabetes Melitus Tipe 2 di Indonesia 2015].[Book in
Indonesian]. Jakarta: Indonesian Society of Endocrinology; 2015.

12. Seaquist ER, Anderson J, Childs B, et al. Hypoglycemia and diabetes: A report of a workgroup of the American Diabetes Association and the Endocrine Society. Diabetes Care 2013;36:1384-95.

13. Riddle M. Updates to the standards of medical care in diabetes2018. Diabetes Care 2018;41:2045-7.

14. Evert AB. Treatment of mild hypoglycemia. Diabetes Spectr 2014;27:58-62.

15. International Diabetes Federation. Global guideline for type 2 diabetes. Brussels: International Diabetes Federation; 2012.

16. Wibisono AH, Lestari AN, Sorensen L, et al. Fear of injections among people with type 2 diabetes: Overview of the problem. J Diabetes Nurs 2017;21:91-5.

17. Coyle ME, Francis K, Chapman Y. Self-management activities in diabetes care: A systematic review. Aust Heal Rev 2013;37:513-22.

18. Royal Australian College of General Practitioner. Chronic condition self-management guidelines summary for nurses and allied health professionals. Sydney: Royal Australian College of General Practitioner; 2015.

19. LaManna J, Litchman ML, Dickinson JK, et al. Diabetes education impact on hypoglycemia outcomes: A systematic review of evidence and gaps in the literature. Diabetes Educ 2019;45:349-69.

20. Kavuran E, Yurttas A. Effects of education based on Roy adaptation model on diabetes care profile of patients with type 2 diabetes. Iran Red Crescent Med J 2018;20:e64635.

21. Herdman T, Kamitsuru S. Nursing diagnoses definitions and classification 2018-2020. 11th ed. New York: Thieme Publishers; 2018.

22. Bulechek G, Butcher H, Dochterman J, et al. Nursing interventions classification. 6th ed. St. Louis: Elsevier Mosby; 2013.

23. Aligood M. Nursing theorists and their work. Maryland Heights: Elsevier Mosby; 2014.

24. Roy S, Andrews H. The Roy adaptation model. Norwalk: Appleton \& Lange; 2009.

25. International Diabetes Federation. Global diabetes plan 20112021. Brussels: International Diabetes Federation; 2011. 